\title{
Frequency Reliance on EEHC-Based Routed Wireless Sensor Networks
}

\author{
Manju Sharma \\ DAV Institute of Engineering \& Technology \\ Jalandhar, Punjab, India
}

\author{
Lalit Awasthi \\ National Institute of Technology \\ Hamirpur, HP, India
}

\begin{abstract}
This paper presents the realistic approach towards the quantitative analysis and simulation of Energy Efficient Hierarchical Cluster (EEHC)-based routing for wireless sensor networks. Here the efforts have been done to combine analytical hardware model with the modified EEHC-based routing model and to investigate the operating frequency reliance on various performance metrics. It has been indicated that there is significant enhancement in the number of cluster requirement as there is increase in frequency and head set size.
\end{abstract}

\section{Keywords}

EEHC, LEACH, Wireless Sensor Networks, Energy Consumption.

\section{INTRODUCTION}

The basic element of a wireless sensor networks (WSN) is the sensor node, where it is consisted of three main functional components that separately deliver sensory, communication and processing capabilities. Because of the difficulty and cost of sensor node replacement in face of battery drainage or system failure, hardware reliability is also another major concern in WSN design. Therefore keeping in realistic facts and operational conditions the focus of this paper is report the realistic quantitative analysis EEHC-based routing for WSN.

Many energy-efficient routing protocols are designed based on the clustering structure. The randomized clustering algorithm to organize sensors into clusters in a WSN was proposed Bandyopadhyay and Coyle [1]. Further the computation of the optimal probability of becoming a cluster head was presented. In [2], Moscibroda and Wattenhofer defined the maximum clusterlifetime problem, and they proposed distributed, randomized algorithms that approximate the optimal solution to maximize the lifetime of dominating sets on WSNs.

Low-energy adaptive clustering hierarchy (LEACH) [3] is one of the first hierarchal routing approaches for WSNs. It is a wellknown clustering protocol for wireless sensor networks and most of the clustering algorithms are based on this algorithm. This protocol uses only two layers for communication. LEACH includes distributed cluster formation, local processing to reduce global communication, and randomized rotation of cluster-heads. In literature it has been reported that $\mathrm{LEACH}$ performs over a factor of 7 reductions in energy dissipation compared to flat base routing algorithm such as direct diffusion [4]. But the main problem with LEACH protocol lies in the random selection of cluster heads. There exists a probability that the cluster heads formed are unbalanced and may remain in one part of the network making some part of the network unreachable.

As an extension of LEACH [3, 5-7], the proposed protocol introduces a head set for the control and management of clusters. Although S-MAC [8] divides the network into virtual clusters, the proposed protocol divides the network into a few real clusters that are managed by a virtual cluster-head. The results reported in this paper are the efforts towards the realistic approach by extending of work reported in [7, 9-10]. The derivations for transceiver power consumption modeled in [9] and [7] are considered to report modified quantitative analysis for the EEHC based routing for WSN. This paper is extension of paper [10].

The rest of this paper is organized as follows. After mentioning the introduction in section 1 , the proposed model is discussed in section 2. In Section 3, we have described the quantitative analysis for EEHC-based WSN. In section 4 the results have been shown and described based on operating frequency EEHC via simulations. Finally, Section 5 concludes the paper.

\section{PROPOSED MODEL}

The proposed routing scheme is based on the fact that the energy consumed to send a message to a distant node is far greater than the energy needed for a short range transmission. Here a simple radio frequency (RF) transceiver model (Figure 1) has been considered that is connected to the sensing and processing unit of the sensor node. Data to be transmitted will first pass through the digital-to-analog converter (DAC) and low-pass filter to prepare for up-conversion at the mixer with carrier signal generated by the frequency synthesizer. The subsequent modulated signal will be transmitted by the power amplifier (PA) over the wireless channel.

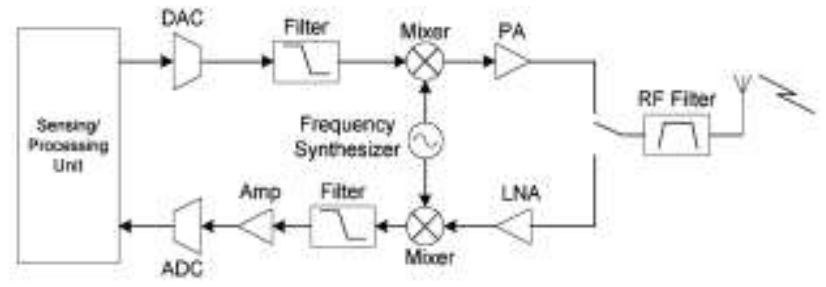

Figure 1 A simple RF transceiver model of sensor node

On the receive path, incoming signals will first be amplified by the low noise amplifier (LNA), and then demodulated and processed through a series of intermediate frequency (IF) and base-band filters and amplifiers (not explicitly shown in Figure 1 [9]. In the end, digital data are recovered by the analog-to-digital 
converter (ADC) and then forwarded to the processing unit for further decoding.

We have extended the LEACH protocol by using a head-set instead of a cluster head [7] in combination with transceiver model of sensor node [9] and the work reported in [10]. In other words, during each election, a head-set that consists of several nodes is selected. The members of a head-set are responsible for transmitting messages to the distant base station. At one time, only one member of the head-set is active and the remaining head-set members are in sleep mode. The above communication stages are illustrated in [10].

\section{QUANTITATIVE ANALYSIS}

The following derivation for transceiver power consumption is modeled after those given in [9], and has been used later for the analysis. On the transmission path, the total power consumption, $P_{T x}$, can be written as:

$$
P_{T x}=P_{P A}+P_{E}
$$

Where $P_{P A}$ is the amount of power consumed by the $\mathrm{P}_{\mathrm{A}}$ alone, and $P_{E}$ is the amount of power collectively consumed by the other electronic components such as the mixer, frequency synthesizer, DAC, and various filters. Determining the exact values for both $P_{P A}$ and $P_{E}$ would depend on RF component design and device technology, which is beyond the scope of this research; though a simple approximation would suffice in the current work. While $P_{E}$ is generally treated as a constant under various operating conditions, $P_{P A}$ can be further broken down into the following terms:

$$
P_{P A}=\frac{P_{R x S i}\left(\frac{1}{L_{o}}\right)\left(\frac{R_{c o m}}{d_{o}}\right)^{\alpha}}{G_{T x} G_{R x} \eta}
$$

Where $P_{R x S i}$ denotes the receiver sensitivity in Watts, $L_{o}$ is the path loss attenuation at $d_{o}$ metres, $R_{\text {com }}$ refers to the distance between the transmitter and the receiver in metres, $\alpha$ is the path loss exponent, $G_{T x}$ and $G_{R x}$ represent transmit and receive antenna gains, respectively, and $\eta$ stands for PA efficiency. In turn, the receiver sensitivity $P_{R x S i}$ can be rewritten as[10]:

$$
P_{R x S i}=\left(\frac{S}{N}\right)_{R x} \cdot N F_{R x} \cdot N_{o} \cdot B W
$$

Where $\left(\frac{S}{N}\right)_{R x}$ is the minimum signal-to-noise ratio that provides an acceptable $\left(\frac{E_{b}}{N_{o}}\right)$ level at the receiver, $N_{F R x}$ is the noise factor at the receiver, $N o$ is the thermal noise floor in a 1 $\mathrm{Hz}$ bandwidth (in $\mathrm{W} / \mathrm{Hz}$ or $\mathrm{J}$ ), and $B W$ is the channel noise bandwidth (in $\mathrm{Hz}$ ). Let:

$$
\frac{1}{L_{o}}=\left(\frac{4 \pi}{w}\right)^{\alpha}, d_{o}=0.1
$$

Where $w$ is the wavelength of the carrier frequency in meters. Substituting Eq.s (3) and (4) into (2), $P_{P A}$ becomes:

$P_{P A}=\frac{\left(\frac{S}{N}\right)_{R x} \cdot N F_{R x} \cdot N_{o} \cdot B W \cdot\left(\frac{4 \pi}{w}\right)^{\alpha} \cdot 10^{\alpha} \cdot d^{\alpha}}{G_{T x} G_{R x} \eta}$

In terms of $\left(\frac{E_{b}}{N_{o}}\right), P_{P A}$ can be represented as:

$P_{P A}=\frac{\left(\frac{E_{b}}{N_{o}}\right)_{R x} \cdot R_{T x} \cdot N F_{R x} \cdot N_{o} \cdot B W \cdot\left(\frac{4 \pi}{w}\right)^{\alpha} \cdot 10^{\alpha} \cdot d^{\alpha}}{G_{T x} G_{R x} \eta}$

On the reception path, the total power consumption, $P_{R x}$, depends on the power consumption of the LNA, mixer, frequency synthesizer, IF amplifiers, filters, and ADC.

Generally the sensor model doesn't specify the transmitting or receiving one bit. Nonetheless, the platform uses transmission rate of $1 \mathrm{Mbps}\left(R_{b i t s}\right)$ or time to send one bit is $1 \mu \mathrm{s}$, so one can calculate the energy required for transmitting one bit, following a method based in the approach presented by Hill et.al in [11]. The energy used in transmitting or receiving one bit and is found by using the power value and can be further derived as:

$\mathcal{E}_{s}=P_{P A} \times$ time $=\frac{P_{P A}}{R_{b i t}}$

Where $R_{b i t}$ is the raw bit rate, time in seconds and power in Watts.

$\varepsilon_{s}=\frac{\left(\frac{S}{N}\right)_{R x} \cdot N F_{R x} \cdot N_{o} \cdot B W \cdot\left(\frac{4 \pi}{w}\right)^{\alpha} \cdot 10^{\alpha} \cdot d^{\alpha}}{G_{T x} G_{R x} \eta R_{b i t}}$ or in terms of $\left(\frac{E_{b}}{N_{o}}\right)$ can be represented as:

$\varepsilon_{s}=\frac{\left(\frac{E_{b}}{N_{o}}\right)_{R x} \cdot R_{T x} \cdot N F_{R x} \cdot N_{o} \cdot B W \cdot\left(\frac{4 \pi}{w}\right)^{\alpha} \cdot 10^{\alpha} \cdot d^{\alpha}}{G_{T x} G_{R x} \eta R_{b i t}}$

The radio communication and energy consumption described in [7] is adopted: for short distance transmission, such as intracluster communication, the energy consumed by a transmitting amplifier is proportional to $d^{2}$ and for long distance transmission, such as inter-cluster communication, the energy consumption is proportional to $d^{4}$. Using the given radio and energy consumption models, the energy consumed in transmitting one message among cluster heads for a distance $d$ is given by

$E_{T}=l E_{e}+l \varepsilon_{l} d^{4}$

Similarly, the energy consumed when the senor node works as a regular (member) node, that is, the energy consumed in 
transmitting a massage within a cluster for a short distance d, is given by

$$
E_{T}=l E_{e}+l \varepsilon_{s} d^{2}
$$

Moreover, the energy consumed to receive the 1-bit message is given by:

$$
E_{T}=l E_{e}+l E_{B F}
$$

Eq. 11 includes the cost of beam forming approach that reduces energy consumption. The constants used in the radio model are given in Table 1.

Table 1: Parameters values used for radio communication

\begin{tabular}{|c|c|c|}
\hline Description & Symbol & Value \\
\hline $\begin{array}{l}\text { Energy consumed by the } \\
\text { amplifier to transmit at a } \\
\text { longer distance }\end{array}$ & $\varepsilon_{l}$ & $0.0013 \mathrm{pJ} / \mathrm{bit} / \mathrm{m}^{4}$ \\
\hline $\begin{array}{l}\text { Energy consumed in the } \\
\text { electronics circuit to } \\
\text { transmit or receive the } \\
\text { signal }\end{array}$ & $E_{e}$ & $50 \mathrm{~nJ} / \mathrm{bit}$ \\
\hline $\begin{array}{l}\text { Energy consumed for beam } \\
\text { forming }\end{array}$ & $E_{B F}$ & $5 \mathrm{~nJ} / \mathrm{bit}$ \\
\hline Noise factor at the receiver & $N F_{R x}$ & $11 \mathrm{~dB}(12.589)$ \\
\hline $\begin{array}{l}\text { Minimum signal-to-noise } \\
\text { ratio that provides an } \\
\text { acceptable } E_{b} / N_{o} \text { level at the } \\
\text { receiver }\end{array}$ & $\left(\frac{S}{N}\right)_{R x}$ & $10 \mathrm{~dB}(10)$ \\
\hline $\begin{array}{l}\text { Thermal noise floor in a } 1 \\
\mathrm{~Hz} \text { bandwidth (in W/Hz or } \\
\text { J) }\end{array}$ & $N_{o}$ & $\begin{array}{l}-173.8 \mathrm{dBm} / \mathrm{Hz} \\
\left(4.17 \times 10^{-21} \mathrm{~J}\right)\end{array}$ \\
\hline $\begin{array}{l}\text { Wavelength of the carrier } \\
\text { frequency in meters }\end{array}$ & $w$ & $\begin{array}{l}0.328 \mathrm{~m} \text { (for } 915 \\
\mathrm{MHz} \text { ) } \\
0.125 \mathrm{~m} \text { (for } 2.4 \\
\mathrm{GHz})\end{array}$ \\
\hline $\begin{array}{l}\text { Transmit and receive } \\
\text { antenna gains }\end{array}$ & $G_{T x} G_{R x}$ & $-20 \mathrm{dBi}(0.01)$ \\
\hline Stands for PA efficiency & $\eta$ & 0.2 \\
\hline Path loss exponent & $\alpha$ & 2 \\
\hline $\begin{array}{l}\text { Channel noise bandwidth } \\
\text { (in } \mathrm{Hz} \text { ) }\end{array}$ & $B W$ & $1 \mathrm{bit} / \mathrm{Hz} \times \mathrm{B}_{\mathrm{Tx}}$ \\
\hline $\begin{array}{l}\text { Amount of power } \\
\text { collectively consumed by } \\
\text { the other electronic } \\
\text { components such as the } \\
\text { mixer, frequency } \\
\text { synthesizer, DAC, and } \\
\text { various filters }\end{array}$ & $P_{E}$ & $3.63 \mathrm{~mW}$ \\
\hline
\end{tabular}
model and other for quantitative analysis
There is uniform distribution of clusters and each cluster contains $n / k$ nodes. For a sensor network of $n$ nodes, the optimal number of clusters is given as $k$. It is assumed that:

The quantitative analysis is based on the radio model for shorter distance Eq. 9 and keeping all assumptions as same mentioned in [10]. For each cluster, the corresponding cluster head chooses a set of $m$ associates, based on signal analysis.

\subsection{Election phase}

Using Eq. 8, 10 and Eq. 11, the energy consumed by a cluster head is estimated as follows:

$$
\begin{aligned}
E_{C H-\text { elec }} & =\left\{l E_{e}+l \frac{\left(\frac{E_{b}}{N_{o}}\right)_{R x} \cdot R_{T x} \cdot N F_{R x} \cdot N_{o} \cdot B W \cdot\left(\frac{4 \pi}{w}\right)^{\alpha} \cdot 10^{\alpha} \cdot d^{\alpha}}{G_{T x} G_{R x} \eta R_{b i t}}\right\} \\
& +\left\{\left(\frac{n}{k}-1\right) l \mathbf{E}_{e}+E_{B F}\right\}
\end{aligned}
$$

Similarly the energy consumed by non-cluster head sensor nodes is estimated as follows:

$$
\begin{aligned}
E_{\text {non-CH-elec }}= & \left\{l E_{e}+l \frac{\left(\frac{E_{b}}{N_{o}}\right)_{R x} \cdot R_{T x} \cdot N F_{R x} \cdot N_{o} \cdot B W \cdot\left(\frac{4 \pi}{w}\right)^{\alpha} \cdot 10^{\alpha} \cdot d^{\alpha}}{G_{T x} G_{R x} \eta R_{b i t}}\right\} \\
& +k l \mathbf{E}_{e}+E_{B F}
\end{aligned}
$$

\subsection{Data transfer phase}

As mentioned in [7] during data transfer phase, the nodes transmit messages to their cluster head and cluster heads transmit an aggregated messages to a distant base station. The energy consumed by a cluster head is as follows:

$$
E_{C H / \text { frame }}=l E_{e}+l \varepsilon_{l} d^{4}+\left\{\left(\frac{n}{k}-m\right) l \mathbf{E}_{e}+E_{B F}\right\}
$$

The first part of Eq. 14 shows the energy consumed to transmit a message to the distant base station. The second part of Eq. 14 shows the energy consumed to receive messages from the remaining $\left(\frac{n}{k}-m\right)$ nodes that are not part of the head-set.

The energy, $E_{n o n-C H}$ / frame , consumed by a non-cluster head node to transmit the sensor data to the cluster head is given below:

$E_{\text {non-CH / frame }}=l E_{e}+l \frac{\left(\frac{E_{b}}{N_{o}}\right)_{R x} \cdot R_{T x} \cdot N F_{R x} \cdot N_{o} \cdot B W \cdot\left(\frac{4 \pi}{w}\right)^{\alpha} \cdot 10^{\alpha} \cdot d^{\alpha}}{G_{T x} G_{R x} \eta R_{b i t}}$

The energy consumptions in a data transfer stage of each cluster are as follows:

$E_{\text {CH-data }}=f_{1} N_{f} E_{C H / \text { frame }}$ 
$E_{\text {non-CH-data }}=f_{2} N_{f} E_{\text {non_CH / frame }}$

Where $f_{1}=\left(\frac{1}{\frac{n}{k}-m+1}\right) \cdot \frac{1}{k} \quad \& \quad f_{2}=\left(\frac{\frac{n}{k}-m}{\frac{n}{k}-m+1}\right) \cdot \frac{1}{k}$

\subsection{Starting energy for one round}

The start energy, $E_{\text {start }}$, is energy of a sensor node at the initial start time. This energy should be sufficient for at least one round. In one round, a node becomes a member of head-set for one time and a non-cluster head for $\left(\frac{n}{k m}-1\right)$ times. An estimation of $E_{\text {start }}$ is given below:

$E_{\text {start }}=\frac{E_{C H-\text { elec }}+E_{\text {non-CH-elec }}}{m}+\frac{N_{f}}{m} \boldsymbol{\varphi}_{1} E_{C H / \text { frame }}+f_{2} E_{\text {non-CH / frame - }}$ -

and from this Eq. $N_{f}$ can be derived as:

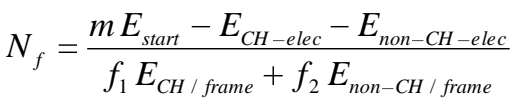

Here it has been assumed that there are $k$ clusters and $n$ nodes. In each iteration, $m$ nodes are elected for each cluster. Thus, in each iteration $k m$ nodes are elected as members of head-sets. The number of iterations required for all $n$ nodes to be elected is $\left(\frac{n}{k m}\right)$, which is the number of iterations required in one round. Moreover, an iteration consists of an election phase and a data transfer stage.

\subsection{Optimum number of clusters}

Following [7] and [9] the optimum number of $k$ for minimum consumed energy can be determined as follows:

$$
k=\sqrt{\frac{n}{2 \pi}} \cdot \sqrt{\frac{\left(\frac{E_{b}}{N_{o}}\right)_{R x} \cdot R_{T x} \cdot N F_{R x} \cdot N_{o} \cdot B W \cdot\left(\frac{4 \pi}{w}\right)^{\alpha} \cdot 10^{\alpha} \cdot d^{\alpha}}{G_{T x} G_{R x} \eta R_{b i t}}} \cdot M
$$

\section{RESULTS AND DISCUSSION}

Figure 2 shows a graph that illustrates the variation in the maximum number of clusters size with respect to distance from base station and the head set size at different operating frequencies of $2.4 \mathrm{GHZ}$ and $915 \mathrm{MHZ}$. The graph clearly indicates that there is significant enhancement in the number of cluster requirement as there is increase in frequency and head set size. This has been indicated by the figure 2 that the requirement of number of clusters reduces at smaller head set size and distance.

The maximum number of clusters at head set size of $m=3$ with different frequencies of sensor nodes has been indicated in figure 3 . Here the figure 3 depicts that there is increase in requirement of energy as operating frequencies increases. The energy requirement becomes equal at if optimum number of clusters selected is 7 at head set size of 3 . Therefore the frequency impact is significant for less number of clusters.

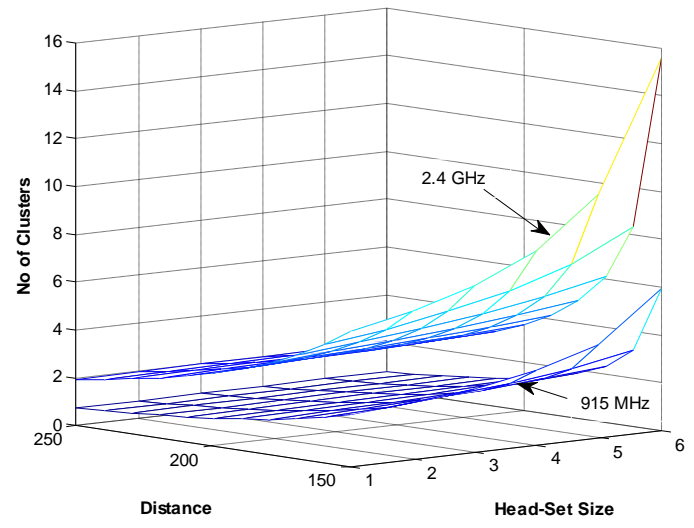

Figure 2 Cluster size with respect to distance from the base station and the headset size at $2.4 \mathrm{GHz}$ and $915 \mathrm{MHz}$.

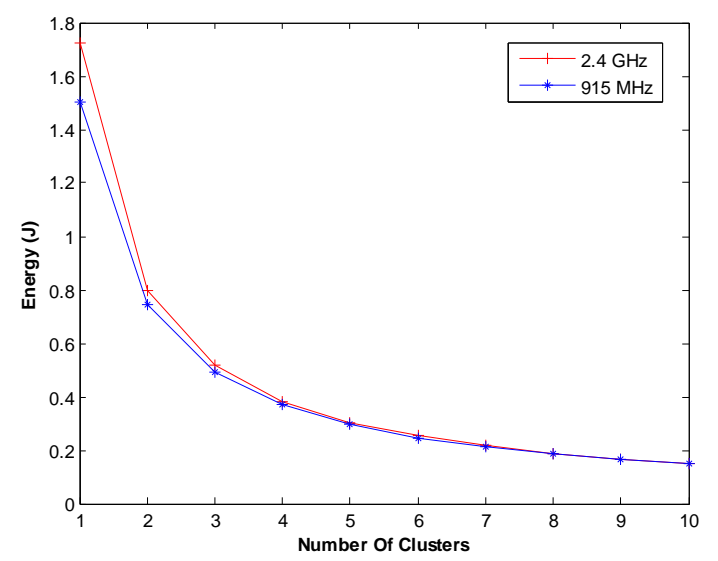

Figure 3 Maximum optimum number of clusters at Head-set size of $\mathbf{m}=\mathbf{3}$ with different frequencies of sensor node

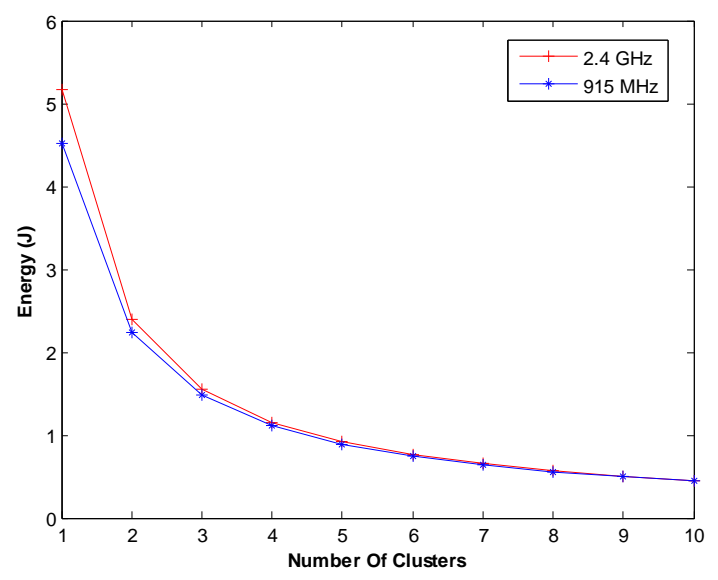

Figure 4: Energy consumption at the maximum optimum number of clusters at Head-set size of $m=1$ with different frequencies of sensor node 
Figures 3 and 4 illustrates that the energy consumption is much more dependent upon the number of clusters. The figure clearly indicates that for head set size of $m=1$ the energy consumption rises significantly in comparison with $\mathrm{m}=3$ (figure 3 ). It is [4.5, 5.2] and [1.5, 1.7] ( $\mathrm{J})$ in case of $915 \mathrm{MHZ}$ and $2.4 \mathrm{GHZ}$ respectively.

\section{CONCLUSIONS}

This paper presents the observations obtained from the combination of realistic analytical wireless sensor model and EEHC-based routing for wireless sensor networks for operating frequency dependence. Here it is illustrated that the variation in the maximum number of clusters size with respect to distance from base station and the head set size at different operating frequencies of $2.4 \mathrm{GHz}$ and $915 \mathrm{MHz}$ is of importance. It has been shown that there is significant enhancement in the number of cluster requirement as there is increase in frequency and head set size. Results also depicts that there is increase in requirement of energy as operating frequencies increases. The energy requirement becomes equal at if optimum number of clusters selected is 7 at head set size of 3 .

\section{REFERENCES}

[1] S. Bandyopadhyay, E.J. Coyle, An energy-efficient hierarchical clustering algorithm for wireless sensor networks, in: IEEE INFOCOM, vol. 3, pp. 1713-1723, 2003.

[2] T. Moscibroda, R. Wattenhofer, Maximizing the lifetime of dominating sets, in: 19th International Parallel and Distributed Processing Symposium, 2005.

[3] W.R. Heinzelman, A. Chandrakasan, H. Balakrishnan, Energy efficient communication protocol for wireless microsensor networks, in: Hawaii International Conference on System Sciences (HICSS), 2000.
[4] Govindan, R., and Intanagonwiwat, D. E. C., Directed diffusion: a scalable and robust communication paradigm for sensor networks. In Proceedings of the 6th annual ACM/IEEE international conference on mobile computing and networking, pp. 56-67, 2000.

[5] W. Heinzelman, Application-specific protocol architectures for wireless networks, Ph.D. thesis, Massachusetts Institute of Technology, 2000.

[6] W. R. Heinzelman, A. P. Chandrakasan, H. Balakrishnan, An application-specific protocol architecture for wireless microsensor networks, IEEE Transactions on Wireless Communications, vol. 1 (4) 660-670, 2002.

[7] S. Hussain and A. W. Matin, "Energy Efficient Hierarchical Cluster-Based Routing for Wireless Sensor Networks", Jodrey School of Computer Science Acadia University Wolfville, Nova Scotia, Canada, Technical Report, pp. 1-33, 2005.

[8] W. Ye, J. Heidemann, and D. Estrin. Medium access control with coordinated adaptive sleeping for wireless sensor networks. IEEE/ACM Transactions on Networks, vol. 12(3), pp. 493-506, 2004.

[9] R. W. K. Ha, “A Sleep-Scheduling-Based Cross-Layer Design Approach for Application-Specific Wireless Sensor Networks" Ph.D. thesis University of Waterloo, Ontario, Canada, pp. 22-24, 2006.

[10] Manju Sharma and Lalit Awasthi, "Realistic Approach towards Quantitative Analysis and Simulation of EEHCBased Routing for Wireless Sensor Networks", IJCSI International Journal of Computer Science Issues, Vol. 7, Issue 1, 2010.

[11] J. Hill, R. Szewczyk, A. Woo, S. Hollar, D. Culler, K. Pister. "Architecture Directions for Networked Sensors", ASPLOSIX 11/00 Cambridge, MA, USA, Nov. 2000. 